\title{
Recursos materiales para la prevención y el tratamiento de las úlceras por presión: análisis de la situación en Andalucía
}

\section{Resources for prevention and treatment of pressure ulcers: the situation in Andalusia (Spain)}

Francisco Pedro García Fernández

Pedro Luis Pancorbo Hidalgo

Jesús López Ortega

Isabel María López Medina

\author{
Diplomado en Enfermería. Unidad de Formación, Investigación y Calidad. Complejo \\ Hospitalario de Jaén. \\ Doctor en Biología. Diplomado en Enfermería. Departamento de Ciencias de la Salud. \\ Universidad de Jaén. \\ Bachelor in Nursing. Diplomado en Enfermería. Departamento de Ciencias de la Salud. \\ Universidad de Jaén. \\ Licenciada en Antropología Social y Cultural. Diplomada en Enfermería. Departamento \\ de Ciencias de la Salud. Universidad de Jaén. \\ Miembros del Grupo de investigación Enfermería.
}

Correspondencia:

Francisco Pedro García Fernández

Unidad de Formación, Investigación y Calidad

Complejo Hospitalario de Jaén

Avda. del Ejercito Español, no 10

23007-Jaén

E-mail: fpgarciaf23@enfermundi.com

Este estudio fue realizado con ayuda económica a la investigación del III Plan Andaluz de Investigación (Consejería de Educación y Ciencia de la Junta de Andalucía) al Grupo de investigación "Enfermería” CTS 464. 
Objetivos: Cuantificar la disponibilidad de medios materiales de prevención y tratamiento de úlceras por presión (UPP) utilizados en la práctica profesional enfermera en los centros sanitarios y sociosanitarios andaluces. Métodos: Mediante muestreo aleatorio estratificado se incluyeron en el estudio 11 hospitales, 36 centros de salud de Atención Primaria y 14 residencias sociosanitarias geriátricas de la red pública de Andalucía. Se utilizó un cuestionario autocumplimentado enviado a los responsables de enfermería de las unidades o centros incluidos, con preguntas sobre indicadores epidemiológicos, protocolos, escalas de valoración del riesgo, documentación específica, materiales de prevención y productos para el tratamiento local de UPP. Se realizó un análisis descriptivo de los resultados. Resultados: Se obtuvieron 60 cuestionarios válidos (tasa de respuesta del 65,9\%). El 100\% de las residencias geriátricas, el 96,4\% de las unidades hospitalarias y el 9,5\% de los centros de salud disponen de alguna unidad de superficie especial de alivio de la presión (SEMP). Los centros mejor dotados de este material preventivo son las residencias geriátricas (disponen de SEMP estáticas y dinámicas, para cama y para sillón); las unidades hospitalarias tienen una dotación muy variable según hospital; mientras que en los centros de salud carecen, en la práctica, de este material. El tipo más usual de SEMP son colchones y colchonetas estáticas, junto a colchonetas de aire. Los productos preventivos locales más usados son: leches hidratantes y ácidos grasos hiperoxigenados, aunque en algunos centros continúa usándose la povidona yodada con este fin. Con respecto al tratamiento local de las UPP, mayoritariamente, es la enfermera que atiende al paciente quien determina el producto a utilizar. Para la limpieza de la úlcera casi todos los centros citan el suero salino; sin embargo, un porcentaje importante de centros considera también diversos antisépticos como productos de limpieza de UPP. La gama de apósitos y productos disponibles para el tratamiento de UPP es más amplia en las residencias geriátricas (8 productos como media) que en los hospitales (media de 4 productos). Los productos disponibles en mayor número de centros son: hidrocoloides, hidrogeles, antibióticos tópicos, pomadas cicatrizantes y pomadas enzimáticas. Conclusiones: La disponibilidad de recursos de prevención, como SEMP, es buena en las residencias geriátricas, pero insuficiente, tanto en cantidad como en tipos de las SEMP, en muchas unidades hospitalarias. Los centros de salud no disponen de este material. Los productos locales para la prevención y el tratamiento de las UPP disponibles en los hospitales y residencias geriátricas están bastante bien adaptados a las recomendaciones actuales de las guías de práctica clínica; no obstante, persiste, aunque en pocos centros, la utilización de antisépticos para la prevención o limpieza de UPP. Todos los centros disponen de productos para la cura húmeda de heridas. Los tipos de apósitos que llevan más tiempo comercializados están presentes en mayor número de centros.
La calidad de la práctica clínica en la prevención y el tratamiento de las úlceras por presión (UPP) se ve influida fundamentalmente por tres factores (1): a) Un conocimiento adecuado de las guías de práctica clínica más actuales; b) Suficiente motivación para aplicarlas; c) Disponibilidad de los recursos materiales necesarios. Este último aspecto, el de la disponibilidad y adecuación a las necesidades de materiales específicos para la prevención de UPP, constituye un punto clave en los programas preventivos, porque la falta de material o su escasa disponibilidad es identificada por los profesionales de enfermería como una de las principales causas, junto con la falta de personal, que dificultan la aplicación de unos cuidados de calidad en la prevención y tratamiento de UPP (2-5).

Máxime cuando hoy sabemos, como determinan algunos investigadores como Pam Hibbs (citada por Waterlow (6)), que hasta el 95\% de las lesiones es evitable con los cuidados y los recursos adecuados, y éste es un elemento muy a tener en cuenta puesto que el mejor tratamiento de las úlceras por presión es su prevención, no sólo por las complicaciones derivadas para el paciente, sino también para los servicios de salud, donde diariamente en España se atiende a 62.800 personas con UPP en los diferentes niveles asistenciales (7), con un coste estipulado en torno a los $1.687 \mathrm{mi}$ llones de euros (más de 280.000 millones de las antiguas pesetas).

Sin embargo y a pesar de su importancia, los estudios destinados a 


\section{PALABRAS CLAVE}

Úlceras por presión, práctica clínica, superficies especiales para el manejo de la presión, apósitos, tratamiento local.

\section{SUMMARY}

Objectives: To quantify the availability of material resources for pressure ulcers (PU) prevention and treatment in health care centres from Andalusia (Spain). Methods: Eleven hospitals, 36 primary care centres and 14 nursing bomes were included by random stratified sampling. All the centres belonged to the public health or social services of Andalusia (Spain). A self-administered questionnaire was send out to nursing managers of the units or centres. The questionnaire has questions about epidemiological index, protocols, risk assessment scales, specific record use and materials and products for prevention and local treatment of PU. A descriptive analysis of the results was carried out.

Results: Sixty valid questionnaires were obtained (response rate was 65,9\%). A $100 \%$ of the nursing homes, a 96,4\% of the hospital units and a 9,5\% of the primary care centres have some unit of pressure-relief surfaces (PRS). Nursing bomes are the best equipped centres (with static and dynamic PRS, and also for bed and chair); in hospital units the equipment is very variable between hospitals; while primary care centres lack this devices. The most usual PRS are static mattresses and overlays and air overlays. Products for local prevention most commonly used are: moisturizing milks and hyper-oxygenated fatty acids; although some centres are still using iodine povidone for this purpose. On the matter of PU treatment, the nurse who care the patient mostly decides the product to use. For cleaning the ulcer almost all the centres have the saline solution, however an important number of centres consider also several antiseptics as $P U$ cleaning products. The number of dressings and products available is higher in nursing homes (8 products as average) than in hospitals (4 products as average). Products and dressing available in most centres are: hydrocolloids, hydrogels, topical antibiotics, healing cream and enzymatic cream. Conclusions: The availability of prevention resources, such as PRS, is good in nursing homes, but insufficient, in quantity and in kind of support surfaces, in many hospital units. Primary care centres have not this devices. Products for PU prevention and treatment available in hospitals and nursing homes fit in with guidelines current recommendations; nevertheless, antiseptics are still been used for PU prevention and cleaning in some few centres. All the centres have dressings for treatment of wounds in moist environment. The types of dressings first marketed are present in more centres. The range of dressings available in hospitals is small, whereas in nursing homes is wider.

\section{KEY WORDS}

Pressure ulcer, clinical practice, pressure-relief surface, dressing, treatment.

evaluar la dotación de medios materiales preventivos y de tratamiento en los centros sanitarios son muy escasos, tanto en nuestro país (8-10) como en países de nuestro entorno $(4,11,12)$, lo cual hace difícil contrastar con datos objetivos la percepción de baja disponibilidad expresada por las enfermeras clínicas, cuya importancia ha sido puesta de manifiesto por diversos autores, tanto en hospitales $(9,10,13,14)$ como en la atención de salud comunitaria $(15,16)$, donde han demostrado la mejora en los indicadores epidemiológicos de UPP relacionada con el aumento de la disponibilidad de superficies especiales de manejo de la presión (SEMP).

Este trabajo se engloba dentro de un proyecto de investigación destinado a establecer el grado de conocimiento que tienen las enfermeras de las recomendaciones contenidas en las guías de práctica clínica sobre prevención y tratamiento de las UPP y el grado de utilización en la práctica asistencial, en el que también nos preguntábamos por el uso de protocolos y escalas de valoración, el grado documentación de los cuidados, así como la disponibilidad de recursos para la prevención y el tratamiento, centrado el estudio en los centros sanitarios públicos de Andalucía (hospitales, centros de salud y residencias geriátricas o sociosanitarias asistidas).

\section{OBJETIVOS}

Cuantificar la disponibilidad de medios materiales de prevención y tratamiento de UPP utilizados en la práctica profesional enfermera en los 
50 centros sanitarios y sociosanitarios andaluces.

\section{MATERIAL Y MÉTODO}

El período de recogida de datos del estudio comprendió desde junio de 2001 hasta diciembre de 2002. El estudio se centró en los centros sanitarios y sociosanitarios de la red andaluza. Esta red comprende 33 hospitales públicos y 268 centros de salud de Atención Primaria, dependientes de la Consejería de Salud de la Junta de Andalucía. También hemos considerado las 103 residencias geriátricas propias o concertadas por el Instituto Andaluz de Servicios Sociales que cuentan con camas para pacientes asistidos. Previamente al muestreo, realizamos la estratificación de los hospitales y las residencias geriátricas en función de su tamaño, estableciendo dos grupos: hospitales de más de 500 camas (13 en total) y de menos de 500 camas (20 centros); y residencias geriátricas de más de 140 camas asistidas (7 centros) y de menos de 140 camas (95 centros).

A partir del tamaño de muestra calculado para el estudio principal (error $\alpha=0,01$; error $\beta=0,01$; precisión $=3 \%$ ) se determinó el número de centros a incluir en el estudio. El muestreo se realizó de forma aleatoria a partir de un listado numerado de toda la población de hospitales, centros de salud y residencias geriátricas. Fueron seleccionados 11 hospitales ( 4 de más de 500 camas y 7 de menos), 36 centros de salud y 14 residencias geriátricas (4 de más de 140 camas asistidas y 10 menores).

\section{Diseño}

El estudio se realizó mediante un cuestionario autocumplimentado que se envió por vía postal a los responsables de enfermería de los centros y unidades participantes, concretamente a las adjuntas de enfermería en los centros de salud; las supervisoras en las unidades hospitalarias y los responsables asistenciales en las residencias geriátricas. Previamente al envío del cuestionario se estableció un contacto telefónico con los responsables del centro para informar de la naturaleza del estudio y obtener su conformidad para participar. En cada centro se acordó una persona de referencia para colaborar en el estudio que se encargó de recibir, distribuir y recoger los cuestionarios. Tres semanas después del envío inicial de los cuestionarios se distribuyó una carta de recordatorio para animar a la participación a los centros que no habían respondido.

\section{Instrumentos}

Las variables referidas dentro del cuestionario para el conocimiento de los materiales de prevención y tratamiento incluían ítems sobre:

En los centros de salud:

- Superficies especiales para el manejo de la presión: tipos y cantidad disponible en la unidad o centro.

En hospitales y residencias geriátricas:

- Número de camas o camas asistidas.

- Profesional que establece los productos para el tratamiento local.
- Productos usados para el trata- 61 miento de las UPP: limpieza y tratamiento local.

$\mathrm{El}$ análisis realizado ha sido de tipo descriptivo; las variables cualitativas se expresan mediante distribución de frecuencias y la obtención de los porcentajes correspondientes y las cuantitativas mediante la media y su desviación estándar.

\section{RESULTADOS}

En el presente artículo presentamos los resultados referentes a la disponibilidad y uso de materiales y productos para la prevención y el tratamiento de las UPP, ya que los resultados sobre el uso de protocolos y documentos de registro específicos fueron desarrollados en un artículo previo (1). Obtuvimos 60 cuestionarios válidos de un total de 91 enviados (tasa de respuesta del 65,9\%) procedentes de 28 unidades de hospitalización pertenecientes a 11 hospitales, 11 residencias geriátricas y 21 centros de salud. La distribución de unidades de hospitalización participantes fue: traumatología, 1; cirugía, 6; cuidados intensivos, 9; y medicina interna y hospitalización general, 12 unidades. Entre las residencias geriátricas que respondieron al cuestionario, 2 superaban las 100 camas para pacientes asistidos, siendo el resto de menor capacidad.

\section{Superficies especiales \\ de manejo de la presión}

En los hospitales y las residencias geriátricas la situación es buena, ya que disponían de algún tipo de su- 
Tabla 1. Número de centros sanitarios que disponen de materiales de prevención de UPP

\begin{tabular}{|l|l|l|c|}
\hline \multicolumn{4}{|c|}{ Tabla 1. Número de centros sanitarios que disponen de materiales de prevención de UPP } \\
\hline \multicolumn{1}{|c|}{$\begin{array}{c}\text { Materiales de prevención } \\
\text { disponibles }\end{array}$} & $\begin{array}{c}\text { Centros de salud } \\
(\mathbf{n = 2 1 )}\end{array}$ & $\begin{array}{c}\text { Hospitales (unidades } \\
\text { de hospitalización) }(\mathbf{n = 2 8 )}\end{array}$ & $\begin{array}{c}\text { Residencias geriátricas } \\
(\mathbf{n}=\mathbf{1 1})\end{array}$ \\
\hline Protección local (talones, codos, etc.) & $5(23,8 \%)$ & $22(78,6 \%)$ & $11(100 \%)$ \\
SEMP estáticas para cama & 0 & $14(50 \%)$ & $6(54,5 \%)$ \\
SEMP dinámicas para cama & $1(4,7 \%)$ & $19(67 \%)$ & $11(100 \%)$ \\
SEMP estáticas para sillón & $1(4,7 \%)$ & $3(10,7 \%)$ & $9(81,8 \%)$ \\
SEMP dinámicas para sillón & 0 & 0 & 0 \\
\hline SEMP: superficies especiales para el manejo de la presión. & & \\
\hline
\end{tabular}

\section{Tabla 2. Proporción de superficies especiales para el manejo de la presión (SEMP) disponibles de cada tipo y el número de camas de la unidad hospitalaria o de camas asistidas de la residencia}

\begin{tabular}{|c|c|c|}
\hline & $\begin{array}{c}\text { Unidades } \\
\text { de hospitalización* }\end{array}$ & $\begin{array}{c}\text { Residencias } \\
\text { geriátricas** }\end{array}$ \\
\hline Relación no SEMP estáticas/ & Media: $17 \%$ & Media: $50 \%$ \\
camas & Rango: $3-100 \%$ & Rango: $5-100 \%$ \\
Relación no SEMP dinámicas/ & Media: $22 \%$ & Media: $36 \%$ \\
camas & Rango: 3-100\% & Rango: $5-95 \%$ \\
\hline
\end{tabular}

* Seis unidades hospitalarias no indican el número de SEMP estáticas, y 2 unidades no indican el número de SEMP dinámicas, por lo que no se incluyen en el cálculo de la media.

**Dos residencias geriátricas no indican el número de SEMP estáticas, y otras 2 no indican el número de SEMP dinámicas, por lo que no se incluyen en el cálculo de la media.

perficie especial para el manejo de la presión (SEMP) 11 residencias $(100 \%)$ y 27 unidades hospitalarias (96,4\%) (1 unidad no contestó a esta pregunta). En 20 de las unidades de hospitalización las SEMP eran recursos propios de la unidad, mientras que en 7 unidades estaban centralizadas en el hospital para su uso compartido. Sin embargo, en los centros de salud encontramos una importante carencia de estos materiales de prevención, puesto que sólo 2 centros $(9,5 \%)$ dijeron disponer de algún tipo de SEMP.
En cuanto al tipo de SEMP existentes, podemos comprobar en la Tabla 1 que predominan las superficies para utilizar en la cama, mientras que apenas hay disponibilidad de superficies para usar en sedestación. Para determinar la adecuación de la cantidad de estos materiales hemos calculado la proporción entre el número de SEMP y el número de camas de la unidad o centro (Tabla 2). Estos valores indican que en unidades de hospitalización se dispone, en promedio, de una SEMP estática por cada 5,8 camas, y de una SEMP di- námica por cada 4,5 camas. En las residencias las ratios son menores: una SEMP estática cada 2 camas asistidas y una SEMP dinámica cada 2,7 camas asistidas. Cuando examinamos la situación en los diferentes centros, vemos que hay bastante variabilidad, de forma que en unidades de hospital la proporción de SEMP estáticas/cama oscila entre 1:1 (en una unidad de cirugía y una de cuidados intensivos de dos hospitales diferentes); y 1:33 en la unidad con menor proporción. Las ratios de SEMP dinámicas varían entre 1:1 (en una unidad de cuidados intensivos) y 1:33. Las SEMP dinámicas disponibles en los hospitales son de tipo colchoneta de aire de presión alternante en todos los casos, excepto en una unidad de cuidados intensivos en que disponen también de un colchón de aire. Entre las residencias geriátricas también hay mucha variación en las ratios SEMP/cama asistida; oscilando entre 1,24 SEMP estática por cama, en el centro mejor dotado, hasta 1 SEMP estática cada 20 camas en el que dispone de menos recursos; $y$ para las SEMP de tipo dinámico, va- 
52 ría entre $1: 1,05$, y $1: 20 \mathrm{ca}-$ mas. Tres residencias geriátricas disponen de colchones de aire (15, 4 y 4 unidades, respectivamente), mientras que el resto utiliza colchonetas de aire.

\section{Productos locales de prevención}

Para la prevención local de las UPP en zonas de riesgo el producto utilizado en mayor número de centros y unidades de hospitalización son las leches o cremas hidratantes, seguidas por los ácidos grasos hiperoxigenados (AGH), que están bastante introducidos en las unidades de hospitalización, pero no en las residencias geriátricas. Los protectores de barrera cutánea son relativamente poco utilizados. Entre los productos cuya eficacia preventiva no está acreditada vemos que la povidona yodada continúa usándose con esta finalidad en algunos centros y unidades, pero los desinfectantes de tipo colorante, como el mercurocromo y la violeta de genciana, no se usan ya en ninguno de los centros investigados (Tabla 3).

\section{Tratamiento local de las UPP}

El primer aspecto que hemos estudiado es quién decide los productos a utilizar en el tratamiento local de las UPP (Tabla 4). Encontramos

Tabla 3. Productos utilizados para la prevención de UPP sobre piel intacta en las unidades de hospitalización y las residencias geriátricas. Número y porcentaje de unidades que utilizan cada producto

\begin{tabular}{|l|c|c|}
\hline $\begin{array}{l}\text { Productos usados } \\
\text { en la prevención de UPP }\end{array}$ & $\begin{array}{c}\text { Hospitales (unidades } \\
\text { de hospitalización) }(\mathbf{n = 2 8})\end{array}$ & $\begin{array}{c}\text { Residencias geriátricas } \\
(\mathbf{n = 1 1})\end{array}$ \\
\hline Acidos grasos hiperoxigenados & $12(42,9 \%)$ & $2(18,2 \%)$ \\
Protector de barrera cutánea & $4(14,3 \%)$ & $2(18,2 \%)$ \\
Povidona yodada & $2(7,1 \%)$ & $2(18,2 \%)$ \\
Desinfectantes colorantes & 0 & 0 \\
$\quad$ (mercurocromo, etc.) & $18(64,3 \%)$ & $11(100 \%)$ \\
Leches o cremas hidratantes & $1(3,6 \%)$ & $1(9,1 \%)$ \\
Pomada de óxido de zinc & $1(3,6 \%)$ & 0 \\
Vaselina & $2(7,1 \%)$ & 0 \\
Apósito de poliuretano transparente & $7(25 \%)$ & $3(27,2 \%)$ \\
Otros productos (catalasa, aceite & & \\
de almendras, etc.) & & \\
\hline
\end{tabular}

Tabla 4. Responsable de la elección de productos y apósitos para el tratamiento local de UPP en las unidades de hospitalización y residencias geriátricas

\begin{tabular}{|l|c|c|}
\hline $\begin{array}{l}\text { Elección del tratamiento } \\
\text { local de las UPP }\end{array}$ & $\begin{array}{c}\text { Hospitales (unidades } \\
\text { de hospitalización) } \mathbf{( n = 2 8 )}\end{array}$ & $\begin{array}{c}\text { Residencias geriátricas } \\
(\mathbf{n = 1 1})\end{array}$ \\
\hline Enfermera que realiza la cura & $16(78,6 \%)$ & $4(36,4 \%)$ \\
Protocolo & $8(28,6 \%)$ & $2(18,2 \%)$ \\
Supervisora o responsable de unidad & 0 & 0 \\
Médico & 0 & $2(18,2 \%)$ \\
Enfermera + médico & $1(3,6 \%)$ & $1(9,1 \%)$ \\
Otros (enfermera + supervisora, & $2(7,1 \%)$ & $1(9,1 \%)$ \\
$\quad$ varias enfermeras, etc.) & $1(3,6 \%)$ & $1(9,1 \%)$ \\
Sin datos & & \\
\hline
\end{tabular}

que en las unidades hospitalarias existe una gran autonomía de las enfermeras a la hora de decidir este tratamiento, ya que bien directamente o a través de protocolos establecen los productos que se van a utilizar. En ninguna unidad de hospitalización es establecido este tratamiento local por la supervisora de la unidad ni por el médico. En las residencias geriátricas el grado de autonomía en- fermera es algo menor y hay un porcentaje significativo de centros en los que el tratamiento local de las UPP es decidido por el médico. También observamos que el grado de protocolización del tratamiento es bajo.

En respuesta a la pregunta sobre los productos utilizados para la limpieza y/o desinfección de las UPP, hemos encontrado que en casi la to- 
Tabla 5. Productos tópicos que son usados para la limpieza y desinfección de UPP. Número y porcentaje de unidades que utilizan cada producto

\begin{tabular}{|l|c|c|}
\hline $\begin{array}{l}\text { Productos usados en la limpieza } \\
\text { de las UPP }\end{array}$ & $\begin{array}{c}\text { Hospitales (unidades } \\
\text { de hospitalización) (n= 28) }\end{array}$ & $\begin{array}{c}\text { Residencias geriátricas } \\
(\mathbf{n = 1 1})\end{array}$ \\
\hline Suero salino & $27(96,4 \%)$ & $11(100 \%)$ \\
Povidona yodada & $5(17,9 \%)$ & $4(36,4 \%)$ \\
Agua oxigenada & $4(14,3 \%)$ & $4(36,4 \%)$ \\
Clorhexidina & $2(7,1 \%)$ & 0 \\
Jabón & $1(3,6 \%)$ & 0 \\
\hline
\end{tabular}

talidad de unidades hospitalarias y en todas las residencias geriátricas se utiliza el suero salino para este fin (Tabla 5). Sin embargo, continúan empleándose productos de tipo antiséptico, como la povidona yodada, el agua oxigenada o la clorhexidina, que actualmente no se consideran adecuados para la limpieza de UPP, debido a su actividad citotóxica. El porcentaje de centros que usan estos productos es especialmente alto (superior al 60\%) entre las residencias geriátricas.

Actualmente la gama de apósitos y productos para el tratamiento local de las UPP es muy amplia, existiendo productos adaptados a distintos estadios y características de las úlceras. La disponibilidad de estos productos es alta en las residencias geriátricas, donde la media de tipos de productos de tratamiento disponibles en un centro es de $8 \pm 1,41$; en las unidades de hospitalización la gama disponible en cada unidad es algo menor, situándose en 4,8 $\pm 1,98$ tipos de productos. Los productos existentes en mayor número de centros son las pomadas enzimáticas y los apósitos hidrocoloides, seguidos por los hidrogeles. En las residencias geriátricas son muy habituales los antibióticos tópicos, a diferencia de las unidades hospitalarias, en las que están menos presentes. Otros productos de cura tradicional, como las pomadas cicatrizantes y los tules o gasas impregnadas, son más habituales en el ámbito de las residencias geriátricas que en los hospitales. Los apósitos de última generación como los hidrocelulares están introducidos en un porcentaje reducido de hospitales y residencias (Tabla 6).

\section{DISCUSIÓN}

En primer lugar queremos destacar que los datos presentados en este artículo ofrecen una visión de la situación que existe en el sistema sanitario y sociosanitario andaluz con respecto a los medios materiales y recursos para tratar y prevenir las UPP. Nos pareció importante incluir a las residencias geriátricas, ya que en ellas se atiende a gran número de personas con riesgo de presentar UPP y los estudios que las consideran son escasos. Sin embargo, puesto que la estructura de la red de centros sanitarios y sociosanitarios públicos de Andalucía no difiere significativa- mente de la de otras comu- 53 nidades autónomas españolas, pensamos que gran parte de estos resultados podría extrapolarse al ámbito nacional. Sin embargo, serían deseables estudios similares en otras comunidades para comprobar los resultados y conclusiones de esta investigación.

\section{Superficies especiales} para el manejo de la presión

La disponibilidad y adecuación a las necesidades de materiales específicos para la prevención de UPP constituye un punto clave en los programas preventivos. Los resultados de nuestro estudio evidencian que la disponibilidad de superficies especiales para el manejo de la presión es buena en los centros sociosanitarios, moderada (aunque con mucha variabilidad) en los hospitales y casi nula en los centros de salud de Atención Primaria. Las residencias geriátricas disponen de SEMP tanto para cama como para sillón, y para distintos grados de riesgo de UPP, aunque las SEMP de altas prestaciones para pacientes de riesgo elevado están menos introducidas. La dotación de unidades disponibles en función del número de camas asistidas es adecuada en la mayoría de estos centros (un 50\% de camas con SEMP estáticas y un 36\% con SEMP dinámicas). En los hospitales la disponibilidad de estos recursos de prevención es menor. En primer lugar, destacamos la práctica inexistencia de SEMP dinámicas para pacientes de riesgo elevado (tipo colchón de 
Tabla 6. Apósitos y productos tópicos para el tratamiento de UPP. Número y porcentaje de unidades o centros que disponen de cada tipo de producto

\begin{tabular}{|l|c|c|}
\hline $\begin{array}{l}\text { Productos para el tratamiento } \\
\text { de UPP disponibles }\end{array}$ & $\begin{array}{c}\text { Hospitales (unidades } \\
\text { de hospitalización) }(\mathbf{n = 2 8})\end{array}$ & $\begin{array}{c}\text { Residencias geriátricas } \\
(\mathbf{n = 1 1})\end{array}$ \\
\hline Hidrogel & $23(82,1 \%)$ & $9(81,8 \%)$ \\
Hidrocoloide & $25(89,3 \%)$ & $10(90,9 \%)$ \\
Hidrofibras de hidrocoloide & $7(25 \%)$ & $7(63,6 \%)$ \\
Hidrocelulares & $7(25 \%)$ & $4(36,4 \%)$ \\
Alginatos & $7(25 \%)$ & $6(54,5 \%)$ \\
Antibióticos tópicos & $15(53,6 \%)$ & $11(100 \%)$ \\
Tules (gasas impregnadas) & $7(25 \%)$ & $5(45,4 \%)$ \\
Pomadas cicatrizantes & $12(42,8 \%)$ & $10(90,9 \%)$ \\
Apósitos de carbón & $5(17,8 \%)$ & $5(45,4 \%)$ \\
Pomadas enzimáticas & $23(82,1 \%)$ & $11(100 \%)$ \\
\hline
\end{tabular}

reemplazo), incluso en unidades como cuidados intensivos, en las que es frecuente la presencia de pacientes de alto riesgo de UPP, lo que debería ir subsanándose de manera progresiva.

También nos parece importante resaltar que en la mayoría de los hospitales la gestión de estas SEMP la hacen las propias unidades, sólo 4 hospitales tienen un sistema de gestión centralizado de estos recursos. La gestión centralizada de las SEMP ha sido propuesta por varios autores como método para mejorar la eficacia en su utilización (9-17). En segundo lugar, hemos encontrado que las superficies tipo cojín, para prevenir las UPP en los pacientes sentados, están casi totalmente ausentes en los hospitales (sólo 3 unidades disponían de alguna). Esto implica que o bien no se puede movilizar de la cama a los pacientes con riesgo de UPP o con UPP establecidas o bien el tiempo que pasan sentados en sillón no se hace adecuada prevención de estas lesiones. Algunos estudios recientes en otros países han evidenciado un situación similar, aproximadamente un $50 \%$ de enfermeras de hospitales decía tener buena disponibilidad de SEMP $(4,12)$.

Para los pacientes atendidos en sus domicilios por enfermeras de los centros de salud la situación es preocupante, ya que encontramos una falta total de estos recursos básicos para la prevención de UPP. Una investigación reciente en un centro de Atención Primaria que dispone de 17 SEMP concluye que su utilización en pacientes de atención domiciliara con riesgo de UPP disminuye la prevalencia de estas lesiones (15). En el momento de la realización de nuestro estudio, los pacientes en atención domiciliaria que precisaran SEMP para su cuidado debían adquirirlas con sus propios medios, lo cual ha motivado que una mayoría de pacientes y familias no pueda afrontar el gasto económico que ello supone. Esta situación está empezando a cambiar (con 65 posterioridad a nuestra recogida de datos) con la reciente implantación del Decreto de Apoyo a las Familias de Andalucía y la introducción de la enfermera de enlace, que tiene capacidad para gestionar y proporcionar a los pacientes que lo precisen este tipo de recursos de prevención.

\section{Productos para la prevención}

Una proporción alta de unidades de hospitalización, y algo menor de residencias geriátricas, dispone y utiliza productos tópicos para la prevención de UPP adecuados de acuerdo con las recomendaciones de las guías de práctica clínica. Sin embargo, hay algunos aspectos que pueden ser mejorados. Las lociones o cremas hidratantes se encuentran en todas las residencias y la mayoría de las unidades hospitalarias. Los ácidos grasos hiperoxigenados $(\mathrm{AGH})$, cuya efectividad en la prevención e incluso el tratamiento de UPP de estadio I está comprobada (18-20), están presentes en casi la mitad de las unidades hospitalarias, aunque sólo en 2 de las residencias estudiadas. Los protectores cutáneos de barrera están presentes en pocos hospitales y residencias. Varios factores pueden influir en este hecho: por un lado, la reciente aparición de estos productos puede ser el motivo por el que no sean conocidos; por otro, demuestra una falta de actualización de los protocolos de prevención a las 
últimas evidencias científicas. Un aspecto positivo, aunque aún mejorable, es el bajo porcentaje de centros que incluyen la povidona yodada como producto para la prevención, y la aparente desaparición de los desinfectantes colorantes como productos preventivos. Son muy escasos los estudios sobre la utilización clínica de AGH que se encuentran en la literatura científica en inglés (en la base de datos bibliográfica Medline), lo cual nos hace sospechar que este producto aún no es bien conocido en muchos países europeos y de Norteamérica.

\section{Productos para el tratamiento}

En primer lugar, nos parece importante destacar que los resultados que hemos obtenido refuerzan la suposición de que la elección de productos para el tratamiento local de las UPP la hacen las enfermeras que los atienden, en los hospitales y, en menor medida, en las residencias geriátricas. No hemos obtenido esta información en los centros de salud. Esta circunstancia, que no ocurre en otros países de nuestro entorno, ofrece a las enfermeras autonomía y capacidad de decisión en los cuidados locales a pacientes con UPP, pero, al mismo tiempo, supone una importante responsabilidad que obliga a una constante actualización de conocimientos sobre los productos disponibles, sus indicaciones y su utilización correcta.

Con respecto a los productos usados para la limpieza local de las úlceras, hemos comprobado cómo aún persisten productos de tipo desin- fectante (povidona yodada, agua oxigenada, clorhexidina, etc.) que las recomendaciones actuales desaconsejan para esta indicación debido su citotoxicidad (21). El número de unidades hospitalarias y de residencias geriátricas cuyos responsables afirman que se utilizan desinfectantes en la limpieza de UPP nos parece alto y demuestra, una vez más, una falta de diseminación de las recomendaciones basadas en evidencias científicas entre las enfermeras clínicas y gestoras.

Todas las unidades hospitalarias y las residencias geriátricas estudiadas disponían de varios productos para la cura húmeda de heridas. El tipo de apósitos disponibles refleja el orden cronológico de su aparición: así, los apósitos de tipo hidrocoloide están presentes en un mayor número de unidades, mientras que los hidrocelulares, más recientes, están disponibles en una menor proporción de centros. No hemos considerado los apósitos de plata, ya que en el momento del diseño del estudio prácticamente no estaban introducidos en la práctica clínica. Otro punto interesante es la variedad de tipos de apósitos disponibles en una unidad o centro. El proceso de cicatrización de una UPP va a requerir de diferentes tipos de apósitos, en función del estado la misma. Hoy es generalmente aceptado que no puede usarse el mismo tipo de apósito durante todo el tratamiento de una UPP, por tanto, es necesario disponer de una gama adecuada de productos. En este sentido, los resultados de nuestro estudio nos indican una mayor disponibilidad de los distintos tipos de productos de tratamiento en las residencias geriá- 55 tricas (disponen de 8 productos distintos como media), mientras que en las unidades de hospitalización disponen de menor gama (4 tipos como media). Creemos que los sistemas centralizados de adquisición de productos que existen en los hospitales públicos de la red andaluza pueden estar limitando la gama de apósitos disponibles, mientras que en las residencias geriátricas dispondrían de mayor autonomía para la adquisición de los apósitos. Nuevamente una mayor diseminación de las actuales recomendaciones de tratamiento entre los gestores sanitarios, así como la implicación de las enfermeras clínicas en los órganos de decisión de este tipo de material (por ejemplo, a través de las Comisiones de úlceras y heridas crónicas) podría contribuir a solucionar este problema.

\section{Limitaciones}

La investigación tiene algunas limitaciones en su diseño y desarrollo que no podemos dejar de mencionar. En primer lugar, la utilización de un cuestionario autoadministrado que es cumplimentado por los responsables de enfermería de los distintos centros supone que estamos valorando práctica declarada, que puede diferir de la práctica real debido a un sesgo de los informantes para ajustarse a lo que consideran como más correcto. Por tanto, los resultados aquí presentados representan en todo caso la mejor situación y es posible que en algunos aspectos difieran de la realidad. Sin embargo, pensamos que la metodo- 
56 logía que hemos usado nos ofrece una aproximación suficientemente válida a la realidad asistencial de los centros sanitarios y sociosanitarios estudiados. Otra limitación del estudio es la menor cantidad de información que hemos obtenido de los centros de salud, sobre todo referente al tratamiento de las UPP, y que es debida a que en el diseño inicial del cuestionario de recogida de datos no incluimos este aspecto. En las fases posteriores del estudio con los hospitales y las residencias geriátricas el cuestionario fue ampliado con variables sobre el tratamiento. También tenemos que considerar que algunos de los resultados de este estudio pueden haber sufrido modificaciones a partir del año 2002, por la publicación de la Ley de Apoyo a las Familias Andaluzas, que ha supuesto una reforma muy importante dentro de la asistencia enfermera con la creación de la Dirección Regional para el Desarrollo e Innovación de los Cuidados, cuyo primer objetivo ha sido la reforma de la enfermería de Atención Primaria, fundamentalmente con la creación de la figura de la Enfermera Comunitaria de Enlace, con alta capacidad de gestión y provisión de recursos (como SEMP), y la realización de valoraciones integrales de los pacientes en atención domiciliaria, y dentro de ésta la valoración del riesgo de desarrollar úlceras por presión.

\section{CONCLUSIONES}

- La disponibilidad de superficies especiales para el manejo de la presión es variable según el tipo de centro. Una proporción alta de residencias geriátricas está bien dotada de este recurso, la disponibilidad es bastante menor en las unidades hospitalarias y carecen de ellos en los centros de salud.

- El tipo de SEMP más introducido es el tipo colchoneta para pacientes de bajo riesgo. Las superficies tipo colchón, para pacientes de riesgo moderado o alto, están presentes en muy pocos centros.

- La disponibilidad de SEMP para sedestación, tipo cojín, es muy baja en los hospitales.

- La utilización de ácidos grasos hiperoxigenados para la prevención de UPP es muy frecuente en las unidades hospitalarias, pero son poco usados en las residencias geriátricas. Algunos centros continúan usando la povidona yodada para prevenir UPP.

- Las decisiones sobre el tratamiento local de las UPP y la elección del tipo de apósito corresponden, mayoritariamente, a las enfermeras clínicas que atienden a los pacientes.

- Los productos para la cura húmeda de las UPP están introducidos en todos los centros estudiados, aunque la variedad de productos disponibles es mayor en los centros sociosanitarios que en los hospitales.

\section{AGRADECIMIENTOS}

Queremos agradecer su colaboración en el desarrollo de este estudio a las direcciones, supervisoras, adjuntas, enfermeras y auxiliares de enfermería de los siguientes centros sa- nitarios: Hospital Punta Europa de 67 Algeciras (Cádiz), Hospital Alto Guadalquivir de Andújar (Jaén), Hospital de la Axarquía de Vélez-Málaga (Málaga), Hospital de Poniente de El Ejido (Almería), Hospital de La Línea (Cádiz), Hospital Juan Ramón Jiménez (Huelva), Hospital Puerta del Mar (Cádiz), Hospital Virgen Macarena (Sevilla), Hospital de Riotinto (Huelva), Hospital Infanta Margarita de Cabra (Córdoba), Hospital Ciudad de Jaén (Jaén), Centro de Salud (CS) de Iznalloz (Granada), CS de Pozo Alcón (Jaén), CS de Torrox (Málaga), CS de Punta Umbría (Huelva), CS de Cumbres Mayores (Huelva), CS de Santisteban del Puerto (Jaén), CS de Níjar (Almería), CS de Amate (Sevilla), CS Algeciras Norte (Cádiz), CS de Aguilar (Córdoba), CS Polígono Guadalquivir (Córdoba), CS El Ejido (Almería), CS del Puerto de Santa María (Cádiz), CS de Bollullos (Huelva), CS de Fuengirola (Málaga), CS de San Telmo (Cádiz), CS de Benaojan (Málaga), CS Federico del Castillo (Jaén), CS Linamar (Málaga), CS de Puerto Real (Cádiz), CS de Cartaya (Huelva), CS de Isla Cristina (Huelva), Residencia Mixta de pensionistas (Córdoba), Residencia de ancianos La Milagrosa de Armilla (Granada), Residencia mixta de pensionistas de Linares (Jaén), Residencia de la tercera edad de Almería, Centro para mayores Madre de Dios de Almonte (Huelva), Residencia de ancianos Vedruna de Puerto Real (Cádiz), Hospital San Andrés (Córdoba), Residencia Los Ángeles de Fuenteovejuna (Córdoba), Residencia Fuente Salinas de Fuente Vaqueros (Granada), Residencia Santa 
Isabel de Huetor-Tajar (Granada), Residencia San Joaquín y Santa Ana de San Juan del Puerto (Huelva), Re- sidencia Nuestra Sra. de los Dolores de Siles (Jaén), Residencia José María Rivera Paz de Carrión de los Cés- pedes (Sevilla), Residencia Miguel 57 Molinero Martín de Herrera (Sevilla).

\section{BIBLIOGRAFÍA}

1. Pancorbo Hidalgo PL, García Fernández FP, López Medina IMa , López Ortega J. Protocolos y documentación de los cuidados de prevención y tratamiento de las úlceras por presión: análisis de la situación en Andalucía. Gerokomos 2005; 16 (4): 219-28.

2. Wilkes LM, Bostock E, Lovitt L, Dennis G. Nurses' knowledge of pressure ulcer management in elderly people. Br J Nurs 1996; 5 (14): 85865.

3. Provo B, Piacentine L, Dean-Baar S. Practice versus knowledge when it comes to pressure ulcer prevention. J Wound Ostomy Continence Nurs 1997; 24 (5): 265-9.

4. Russell L. Knowledge and practice en pressure area care. Prof Nurse 1996; 11 (5): 301-6.

5. Panagiotopoulou K, Kerr SM. Pressure area care: an exploration of greek nurses' knowledge and practice. J Adv Nurs 2002; 40 (3): 285-96.

6. Waterlow J. Pressure sore prevention manual. Taunton; 1996.

7. Posnett J, Torra i Bou JE. El coste de la atención sanitaria de las úlceras por presión en España. Las úlceras por presión un reto para el sistema de salud y la sociedad (Barcelona/Madrid). 26 y 27 de febrero de 2003. [documento en línea]; disponible en http://www.saludmultimedia.org/congresos/wintertur_gneaupp/posnnett.pdf [Acceso el 29/12/04].

8. Berrade Zubiri E, Luquin Sáinz F. Situación actual de la prevención de las upp en una unidad de Medicina Interna. En: Soldevilla Agreda JJ, Martínez Cuervo F (eds.). Actas del V Simposio nacional sobre úlceras por presión y heridas crónicas. Oviedo: GNEAUPP; 2004.

9. Rodríguez Palma M, Malia Gázquez R, Almozara Molle R, García Pavón F, Gómez Ginéz D, Torra i Bou JE. Impacto de la adquisición de un parque de superficies especiales para el manejo de la presión en la epidemiología de las úlceras por presión en unidades de medicina interna y cuidados paliativos. En: Soldevilla Agreda JJ, Martínez Cuervo F (eds.). Actas del V Simposio nacional sobre úlceras por presión y heridas crónicas. Oviedo: GNEAUPP; 2004.

10. Malia Gámez R, Almozara Molle R, García Pavón F, Ruiz Amaya F, Rodríguez Palma M, Torra i Bou JE. Impacto de la dotación de un parque de superficies especiales para el manejo de la presión en la epidemiología de úlceras por presión de una unidad de cuidados intensivos. En: Soldevilla Agreda JJ, Martínez Cuervo F (eds.). Actas del V Simposio nacional sobre úlceras por presión y heridas crónicas. Oviedo: GNEAUPP; 2004.
11. Torrance C, Maylor M. Pressure sore survey: part one. J Wound Care 1999; 8 (1): 27-30.

12. Sharp C, Burr G, Broadbent M, Casey H, Merriaman A. Pressure ulcer prevention and care: a survey of current practice. J Qual Clin Practice 2000; 20: 150-7.

13. Ramón Cantón C, Salvador Guadayol C, Torra i Bou JE. Úlceras por presión: evaluación de la utilización sistemática de un parque de superficies especiales para el manejo de la presión en la Unidad de Cuidados Intensivos del Hospital de Tarrasa. Enfermería Intensiva 2000; 11 (3): 118-26.

14. Gebhardt KS, Bliss MR, Winwright PL, Thomas J. Pressure-relieving supports in an ICU. J Wound Care 1996; 5 (3): 116-21.

15. Herrero Narváez E, Ordoño Martínez C. Superficies especiales para el manejo de la presión, factor determinante en la disminución de úlceras por presión en atención domiciliaria. En: Soldevilla Agreda JJ, Martínez Cuervo F (eds.). Actas del V Simposio nacional sobre úlceras por presión y heridas crónicas. Oviedo: GNEAUPP; 2004.

16. McKeeney L. Provision of alternating air-pressure mattresses in the community. Nurs Times 2004; 100 (22): 54-6.

17. López Casanova P, Verdú J, Fuentes Pagés G, Ruiz López AL, Donate P, Arias M. Gestión centralizada de un parque de superficies especiales para el manejo de la presión. En: Soldevilla Agreda JJ, Martínez Cuervo F (eds.). Actas del V Simposio nacional sobre úlceras por presión y heridas crónicas. Oviedo: GNEAUPP; 2004.

18. Gallart E, Fuentelsaz C, Vivas G, Garnacho I, Font L, Arán R. Estudio experimental para comprobar la efectividad de los ácidos grasos hiperoxigenados en la prevención de las úlceras por presión en pacientes ingresados. Enfermería Clínica 2001; 11 (5): 179-83.

19. Segovia Gómez T, Bermejo Martínez M, Molina Silva R, Rueda López J, Torra i Bou JE. Cuidados de la piel y úlceras por presión. Rev Rol Enf 2001; 24 (9): 18-22.

20. Torra i Bou JE, Segovia Gómez T, Verdú Soriano J, Nolasco Bonmatí, A, Rueda López, J Arboix i Perejamo, M. Multicentre double blind clinical trial on the effectiveness of Mepentol $^{\circledR}$ a compound of hyperoxigenated fatty acids vs placebo in the prevention of pressure ulcers development. J Wound Care 2005. En prensa.

21. Bergstrom N, Bennett MA, Carlson CE, et al. Treatment of pressure ulcers. Clinical practice guideline, no 15 . Rockville, MD. US. Department of Health and Human Services. Public Health Service. Agency for Health Care Policy and Research; 1994.

\section{INFORMACIÓN PARA LOS AUTORES}

Las NORMAS DE PUBLICACIÓN para todos los interesados en el envío de artículos a la revista Gerokomos pueden encontrarse en las páginas web de la Sociedad Española de Enfermería Geriátrica y Gerontológica http://www.seegg.org, del Grupo Nacional para el Estudio y Asesoramiento en Úlceras por Presión y Heridas Crónicas (http://www.gneaupp.org) o de la propia revista (http://www.drugfarma.com/SPA/gerokomos) 